\title{
La desigualdad en Educación en México por Entidad Federativa 1995 - 2005
}

\section{Inequality in Education by States in Mexico 1995-2005}

\author{
Jesús Salgado Vega \\ Profesor e Investigador \\ Facultad de Economía Universidad Autónoma del Estado de México \\ México \\ jsalgadov@uaemex.mx \\ Karla Grisel Rodríguez Guerra \\ Egresada Facultad de Economía Universidad Autónoma del Estado de México \\ México \\ karla-guerra@hotmail.com
}

Recibido: 20-X-2011 • Aceptado: 27-XI-2011 • Corregido: 15-VI-2012

\begin{abstract}
Resumen: En este artículo se utiliza el coeficiente de Gini para medir la desigualdad en la educación que tienen actualmente los ciudadanos mexicanos en las diferentes entidades del pais, la hipótesis principal es que la desigualdad en educación medida a través del indice de Gini está negativamente asociada con el promedio de años de escolaridad, esto es, que los Estados que han logrado un alto nivel de educación es más probable que logren una mejor igualdad educativa que aquellos que tienen niveles bajos de educación. Según Kutznets, si el nivel de escolaridad se incrementa, la desigualdad en educación primero se incrementa, después llega a un máximo y posteriormente declina. Encontramos que cuando el promedio de los años de estudio en una Entidad Federativa es bajo, tipicamente una pequeña parte de la población tiene todos los años de escolaridad concluidos, y además hay una gran desigualdad educativa entre las entidades o regiones del pais.
\end{abstract}

Palabras clave: Educación, desigualdad, índice de Gini, curva de Kuznets

\section{Introducción}

El acceso equitativo a la educación es una premisa básica de respeto a los derechos humanos, del bienestar presente y futuro de las sociedades. En este artículo se utiliza el coeficiente de Gini para medir la desigualdad educativa que poseen los ciudadanos mexicanos en el periodo en estudio en las diferentes entidades del país. Planteamos la hipótesis principal en la cual la desigualdad en educación está negativamente asociada con el promedio de años de escolaridad, esto es, los estados en donde existe un alto nivel de educación promedio es más probable alcanzar una mejor igualdad educativa entre sus ciudadanos que aquellos con niveles bajos de educación. La desviación estándar ha sido 


\begin{abstract}
In this paper we use the Gini coefficient to measure inequality in education for different states of the country, the main hypothesis is that inequality in education as measured by the Gini index is negatively associated with average years of schooling, that is, States that have achieved a high level of education is more likely to achieve better educational equity than those with low education levels. According to Kutznets, if the level of education increases, the inequality in education first increases, then reaches a maximum and declines thereafter. We found that when the average years of study in one state is low, typically a fraction of Small part of the population has all years of schooling completed, and found also a great educational inequality between the entities or regions of México.
\end{abstract}

Keywords: Education, inequality, Gini index, Kuznets curve utilizada frecuentemente para medir la dispersión absoluta de la distribución de la educación, Birdsall y Londoño (1997) investigaron el impacto de la distribución inicial en el crecimiento y disminución de la pobreza, además encontraron una correlación negativa significativa entre el crecimiento del ingreso y la dispersión en educación.

En el estudio sobre desigualdad en América Latina del Banco Interamericano de Desarrollo (BID) (1999) se utilizó la desviación estándar en la escolaridad y se encontró que cuanto mayor es la desviación estándar, mayor es la desigualdad del ingreso medida por el coeficiente de Gini. Ram (1989) concluyó que si el nivel de escolaridad se incrementa, la desigualdad en educación primero se incrementa, después llega a un máximo y posteriormente declina, tal como la curva de Kuznets, Lim y Kam (2008) estudiaron 99 países y encontraron que la curva de Kuznets en educación se encuentra cuando las medidas de inequidad absoluta son utilizadas y además que la inequidad global en educación ha declinado en las últimas cuatro décadas.

La desviación estándar de la escolaridad mide la dispersión de la distribución de escolaridad en términos absolutos. Para medir la desigualdad relativa de la distribución de la escolaridad es necesario desarrollar una vertiente del índice de Gini aplicado a este fenómeno en particular. Los primeros estudios sobre esta forma de desigualdad relativa empezaron a usar el coeficiente de Gini de la misma manera en que se usaba para medir la distribución del ingreso, la riqueza o la tierra, "cuyo valor varía desde cero, cuando hay perfecta desigualdad, cuando un individuo ostenta toda la riqueza o uno la perfecta igualdad, cuando todos los individuos tienen la misma riqueza” (Salgado, 1983, p. 48). En este artículo se utiliza un índice de Gini de educación diseñado con el objetivo de incluir todos los logros educativos de la población en estudio (López, Thomas y Wang 1999).

La información obtenida para la construcción de los indicadores por 
nivel de escolaridad, para los años de 1995,2000 y 2005 , proviene principalmente de los Anuarios Estadísticos por Entidad Federativa que realiza periódicamente el Instituto Nacional de Geografía y Estadística (INEGI, 1998, 2003 y 2008), es importante señalar que la disponibilidad de datos delimitó el cálculo de los indicadores a un periodo de diez años. México: desigualdad en educación

La desigualdad en México es un fenómeno que sufre al menos el $70 \%$ de la población pues esta presenta algún grado de pobreza derivado de la falta de oportunidades. Es por ello que: "Los gobiernos deberían invertir más dinero en los niños durante sus primeros seis años de vida para reducir la desigualdad social y apoyar a los niños, especialmente a los más vulnerables a tener una vida exitosa y feliz", Organización para la Cooperación y el Desarrollo Económicos OCDE (2010).
Según la evaluación del nivel de formación de los 30 estados integrantes de la OCDE y de otros 27 países, que representan el 90\% del Producto Interno Bruto (PIB) mundial, México ocupó en el 2005 el último lugar de la lista de esos países, este dato además de ser alarmante representa cuatro años de rezago educativo respecto de los países de primer mundo. A continuación se presentan gráficas del 2005 del programa de la OCDE donde se muestran las evaluaciones de estudiantes del Programa Internacional de Evaluación de Estudiantes, PISA (por las siglas en inglés, Program for International Student Assessment) con el fin de ilustrar la situación de México desde una óptica internacional.

Como se observa en la figura 1, el porcentaje de mexicanos entre 25 y 64 años que ha alcanzado una titulación universitaria o de formación profesional superior (15\%) está por debajo del porcentaje que corresponde a

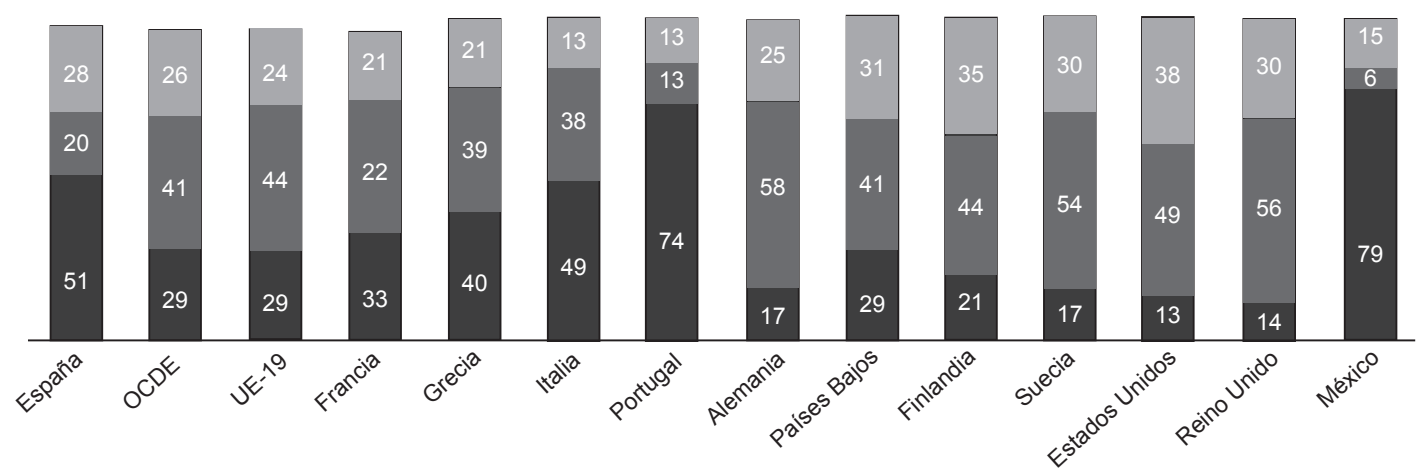

1a. Etapa de educación secundaria

2a. Etapa de educación secundaria

3a. Etapa de educación secundaria

Figura 1. Nivel de formación de la población adulta (25-64 años) (2005)

Fuente: OCDE, 2007 
la media aritmética de la OCDE (26\%), solo el 79\% de la ciudadanía aprobó la primera etapa de educación secundaria lo que equivale en México al nivel básico, de ese modo el nivel de estudios alcanzados es inferior al promedio de esos países. Por lo que se puede concluir que el nivel de estudios alcanzado es inferior al promedio de esos países.

Tabla 1

Población que ha alcanzado el nivel de educación terciaria por grupos de edad (2005)

\begin{tabular}{lccc}
\hline \multicolumn{1}{c}{ País } & años & años & años \\
& $25-54$ & $55-64$ & $25-64$ \\
\hline España & 40 & 14 & 28 \\
OCDE & 32 & 19 & 26 \\
UE-19 & 30 & 17 & 24 \\
Francia & 39 & 16 & 25 \\
Grecia & 25 & 12 & 21 \\
Italia & 16 & 8 & 12 \\
Portugal & 19 & 7 & 13 \\
Alemania & 22 & 23 & 25 \\
Países Bajos & 35 & 24 & 30 \\
Finlandia & 38 & 27 & 35 \\
Suecia & 37 & 25 & 30 \\
Estados & 39 & 37 & 39 \\
Unidos & & & \\
Reino Unido & 35 & 24 & 30 \\
México & 18 & 8 & 15 \\
Brasil & 8 & 4 & 8 \\
Chile & 18 & 9 & 13 \\
Japón & 53 & 22 & 40 \\
\hline
\end{tabular}

Fuente: OCDE, 2007

Como se muestra en la tabla 1 , el porcentaje de 24 a 64 años que completó estudios superiores en el 2005 es de un $15 \%$ posicionando nuevamente al país en los niveles más bajos, por arriba de Brasil y Chile y muy lejos del $40 \%$ de Japón que tiene el porcentaje más alto en ese nivel educativo.
Tabla 2

Distribución del rendimiento de los alumnos en la escala de matemáticas de la OCDE. Pisa (2005)

\begin{tabular}{lc}
\hline \multicolumn{1}{c}{ País } & $\begin{array}{c}\text { Puntuación } \\
\text { Media }\end{array}$ \\
\hline Finlandia & 544 \\
Países Bajos & 538 \\
Japón & 534 \\
Francia & 511 \\
Suecia & 509 \\
Alemania & 503 \\
Castilla y León & 503 \\
País Vasco & 502 \\
Promedio OCDE & 500 \\
Noruega & 495 \\
Cataluña & 494 \\
España & 485 \\
Estados Unidos & 483 \\
Italia & 466 \\
Portugal & 466 \\
Grecia & 445 \\
México & 385 \\
Brasil & 356
\end{tabular}

Fuente: OCDE, 2007

En la tabla 2, se muestra la puntuación media del ranking por países en Matemáticas, México se encuentra en el penúltimo lugar con 385 puntos, dato que revela la notable distancia respecto a los miembros más destacados como Finlandia, Países Bajos y Japón. La OCDE señala que los accesos más equitativos en la educación primaria y secundaria dan como resultado accesos más justos para todas las clases sociales a la educación superior.

El mejor ejemplo de inversión en educación es Finlandia, donde se destina $6 \%$ del PIB, por ello, ha obtenido los mejores resultados en parte, debido a que el nivel de gasto por alumno es mayor al de otros países de alta competencia, 
como, Estados Unidos, Suiza, Noruega, Austria, Islandia, Dinamarca, Italia, Suiza y Francia.

Por el contrario, en el último informe de la OCDE (2011) México es la nación con el gasto público total en educación "más bajo" en relación con el producto interno bruto (PIB). La nación presenta el $24 \%$, y en los demás países el promedio es de $43 \%$.

México se localiza en la segunda posición -solo antes que Turquía- en las tasas de graduación más bajas en enseñanza media superior, con $45 \%$. Además, solo $52 \%$ del alumnado concluye sus estudios en el tiempo estipulado, frente al $68 \%$, en promedio, de las demás naciones. También, tiene el promedio más alto de mujeres de entre 15 y 29 años (38\%) que no están en el sistema educativo y sufren desempleo, en comparación con el de la OCDE, que es de $17.7 \%$.

El gasto por estudiante en México, en todos los casos, se sitúa por debajo de la media en OCDE y coloca a la nación en los últimos lugares. En primaria, la inversión asciende a 2246 dólares, contra 7153 dólares de naciones de la organización; en secundaria es de 2333 dólares, contra 8 972 , y en el nivel superior la cifra es de 7 504 dólares contra 13717.

\section{El problema de la desigualdad}

La desigualdad económica, se entiende como la dispersión de la distribución de los ingresos, en un sistema capitalista como el de México provoca la creación de estratos, niveles o clases sociales donde la desigual distribución de derechos, deberes y responsabilidades que afecta a la mayoría de la población, es otorgada a una minoría que vive en condiciones de privilegio Barro (2000). Al no haber equidad en las regiones algunas quedan rezagadas porque reciben la peor educación, salud e infraestructura quitándoles posibilidades de competitividad en otros mercados, como señala Krugman
(2008), la mejor inversión de un gobierno para disminuir la desigualdad es educar a su población.

La educación es indispensable para desarrollar el capital humano, entendido como una característica intangible que incrementa la productividad de las personas Becker (1967). Las externalidades del capital humano se manifiestan cuando la educación que recibe una persona trabajadora es transmitida a otras, para el caso de México, la desigualdad en educación se deriva de una extensa variedad de factores, pero en este artículo se hace referencia a algunos de ellos, cuya consideración es indispensable.

\section{El capital humano y la educación}

La desigualdad en educación impide al individuo oportunidades de desarrollo Debraj (1998); Schultz (1993), el progreso tecnológico es fundamental en el desarrollo del capital humano puesto que conduce a un cambio dinámico acelerado mediante la retribución de las capacidades laborales de los individuos se transforman cada vez que se completo un ciclo tecnológico en la economía.

El capital humano entendido como el conjunto de las capacidades productivas que un individuo adquiere por acumulación de conocimientos generales o específicos, que pueden ser usados en cualquier sector productivo Becker (1983), dará como resultado a un individuo con miras a aumentar su productividad y rentabilidad, mediante el seguimiento de una formación y capacitación que le permitirán al paso del tiempo percibir salarios más altos y el mantenimiento de su capital psíquico (salud y alimentación), optimizando sus capacidades y evitando que se deprecien pronto, bien por la desvalorización de sus conocimientos generales y específicos o por la degradación de su salud física y moral. 
Para la Organización Internacional del Trabajo (OIT), la educación es un bien imprescindible para el individuo y la sociedad en su conjunto, ya que amplía las posibilidades de acción y elección de las personas y la sociedad en general. En México se han alcanzado importantes logros en las últimas décadas en educación. La cobertura en primaria es casi universal, sin embargo, la Encuesta Nacional de Ocupación y Empleo señala que cerca de 1.7 millones de niños y 1.4 millones de niñas entre 5 y 17 años no asisten a la escuela. Se estima que de la población de 6 a 11 años, en el ámbito nacional, aún no asiste a la escuela entre 1 y $2 \%$ de la población infantil por motivos del trabajo agrícola o debido a impedimentos físicos.
También, en México hay desigualdad en la oferta del servicio que se brinda en las diferentes entidades federativas, en zonas rurales y urbanas, así como en escuelas privadas, públicas y en las escuelas generales e indígenas, de educación comunitaria y para migrantes. En el 2005, 34.9 millones de ciudadanos se identificaron como población en condiciones de rezago educativo; de ellos 17.2 millones tenían la educación primaria, pero no la secundaria; 11.6 millones sabían leer y escribir, pero no contaban con la educación primaria completa; y 6.1 millones eran analfabetas. En los años de 1995 a 2005, el origen del gasto público en educación lo ha aportado en más de un $75 \%$, el gobierno federal, el resto correspondió a los gobiernos estatales, municipales y a particulares.

Tabla 3

Origen del gasto público en educación, 1995-2005 (millones de pesos a precios de 2003)

\begin{tabular}{lcccc}
\hline Año & Total & Federal & Estatal & Municipal \\
\hline 1995 & 242429 & 218043 & 23842 & 544 \\
1996 & 286719 & 335055 & 51132 & 533 \\
1997 & 302211 & 246001 & 55687 & 523 \\
1998 & 3256893 & 267066 & 58078 & 540 \\
1999 & 331810 & 272473 & 58780 & 557 \\
2000 & 354185 & 286796 & 66800 & 589 \\
2001 & 359053 & 289345 & 69114 & 594 \\
2002 & 367851 & 294658 & 72577 & 616 \\
2003 & 386276 & 307407 & 78137 & 733 \\
2004 & 390898 & 308799 & 81319 & 780 \\
2005 & 410074 & 324588 & 84676 & 811 \\
\hline
\end{tabular}

Fuente: INEE, 2007

En la tabla 3 se evidencia un incremento en un periodo de diez años de casi el doble de millones de pesos destinados al gasto público en educación; a pesar de ello, a diferencia de algunos países miembros de la OCDE con mejores calificaciones y promedio más alto de gasto por estudiante, se asocia con un mejor desempeño en las áreas de habilidades; pero, en México el problema de la calidad del sistema educativo quita rentabilidad a esa inversión.

El gasto acumulado por estudiante en México desde el inicio de la educación primaria hasta los 15 años de edad es de 11239 dólares, por debajo del promedio de la OCDE de 43520 dólares. En México, el 
Tabla 4

Años de escolaridad promedio por entidad federativa en México

\begin{tabular}{|c|c|c|c|}
\hline Entidad & $\begin{array}{c}\text { Escolaridad } \\
1995\end{array}$ & $\begin{array}{c}\text { Escolaridad } \\
2000\end{array}$ & $\begin{array}{c}\text { Escolaridad } \\
2005\end{array}$ \\
\hline Aguascalientes & 7.3 & 7.9 & 8.9 \\
\hline Baja California & 7.9 & 8.2 & 9.0 \\
\hline Baja California Sur & 7.9 & 8.4 & 9.0 \\
\hline Campeche & 6.5 & 7.2 & 8.0 \\
\hline Coahuila de Zaragoza & 7.8 & 8.5 & 9.1 \\
\hline Colima & 7.1 & 7.7 & 8.6 \\
\hline Chiapas & 4.8 & 5.6 & 6.2 \\
\hline Chihuahua & 7.3 & 7.8 & 8.4 \\
\hline Distrito Federal & 9.2 & 9.7 & 10.3 \\
\hline Durango & 6.8 & 7.4 & 8.1 \\
\hline Guanajuato & 5.8 & 6.4 & 7.3 \\
\hline Guerrero & 5.6 & 6.3 & 6.9 \\
\hline Hidalgo & 6.0 & 6.7 & 7.6 \\
\hline Jalisco & 7.0 & 7.6 & 8.4 \\
\hline Estado de México & 7.6 & 8.2 & 8.8 \\
\hline Michoacán de Ocampo & 5.8 & 6.4 & 7.0 \\
\hline Morelos & 7.3 & 7.8 & 8.5 \\
\hline Nayarit & 6.7 & 7.3 & 8.2 \\
\hline Nuevo León & 8.4 & 8.9 & 9.6 \\
\hline Oaxaca & 5.1 & 5.8 & 6.5 \\
\hline Puebla & 6.2 & 6.9 & 7.5 \\
\hline Querétaro Arteaga & 6.8 & 7.7 & 8.5 \\
\hline Quintana Roo & 7.1 & 7.9 & 8.6 \\
\hline San Luis Potosí & 6.4 & 7.0 & 7.9 \\
\hline Sinaloa & 7.1 & 7.6 & 8.7 \\
\hline Sonora & 7.8 & 8.2 & 9.0 \\
\hline Tabasco & 6.5 & 7.2 & 8.2 \\
\hline Tamaulipas & 7.5 & 8.1 & 8.8 \\
\hline Tlaxcala & 7.1 & 7.7 & 8.4 \\
\hline Veracruz & 6.0 & 6.6 & 7.3 \\
\hline Yucatán & 6.3 & 6.9 & 7.7 \\
\hline Zacatecas & 5.9 & 6.5 & 7.3 \\
\hline
\end{tabular}

Fuente: INEGI, 1998, 2003 y 2008 
desempeño en la escuela de las personas de 15 años está por debajo del promedio de la OCDE en relación con lo que podría esperarse debido al gasto menor por estudiante.

En la tabla anterior se observa que el comportamiento por estados es equivalente en los tres periodos de estudio, destacando como los mejores el Distrito Federal con un promedio que oscila entre los 9 y 10 seguido por los estados del Norte Nuevo León de 8.5 a 9.5 y finalmente Coahuila y Sonora que presentan de 8 a 9 grados de escolaridad, los últimos cuatro lugares los ocupan los estados del Sur destacando Chiapas con entre 5 a 6 años de escolaridad, Oaxaca entre 6 y 6.5 y Veracruz y Guerrero entre 6.5 a 7 años de escolaridad.

\section{Metodología para el cálculo del índice de Gini en educación}

El índice de Gini es un indicador creado para medir las desigualdades económicas dentro de un país. Indica qué porcentaje de la población accede a los mayores ingresos y, en consecuencia, cuál de ella a los mínimos. Se utiliza para medir la desigualdad en los ingresos, pero puede utilizarse para medir cualquier forma de distribución desigual. El coeficiente de Gini es un número entre 0 y 1 , donde 0 corresponde a la perfecta igualdad (todos tienen los mismos ingresos) y 1 a la perfecta desigualdad (una persona tiene todos los ingresos y los demás ninguno).

Hay dos maneras de calcular el coeficiente Gini, de acuerdo con Deaton (1997) en términos de la distribución de los ingresos, el método indirecto y el directo. En el método indirecto, primero se construye la Curva de Lorenz del ingreso, con el porcentaje acumulado del ingreso sobre el eje vertical y el porcentaje acumulado de la población sobre el eje horizontal. La línea de 45 o es llamada línea de equidistribución ya que representa una sociedad completamente igualitaria en la distribución del ingreso. El coeficiente Gini es calculado como la división de dos áreas, con el área del triángulo de equidistribución como denominador y el área entre la Curva de Lorenz y la línea de equidistribución como numerador. Matemáticamente, el método directo declara que el índice de Gini es la razón de la mitad del promedio sobre todos los pares de las absolutas desviaciones entre todos los posibles pares de personas.

Análogo de la definición de Deaton, aplicado a la educación el índice de Gini mide la razón (promedio de años de educación $\mu$ ) de la mitad del promedio de las desviaciones educativas entre todos los posibles pares de población, para calcularlo se dividió la población en siete categorías; similar a lo hecho por Barro y Lee (1993), que dividen la población en siete categorías incluyendo sin educación (o analfabeta), primaria parcial, primaria completa, secundaria parcial, secundaria completa, terciaria parcial, terciaria completa.

La información referente a los grupos de edad y educación fue obtenida de (INEGI, 1998,2003 y 2008) y que a continuación se describen: la primera $\mathrm{p} 1$, con cero educación, es decir la población de 6 a 14 años que no sabe leer ni escribir la siguiente p2, alumnos de preescolar promovidos, p3, alumnos aprobados en primaria $\mathrm{p} 4$, alumnos aprobados en secundaria p5, alumnos aprobados en bachillerato al final del curso, p6 alumnos inscritos en licenciatura y, por último p7 alumnos inscritos en postgrado. Las yi corresponden a los años de estudio de la población, para aquellos que nunca han asistido a la escuela y1 igual a cero, y2 los que tienen

$E_{L}=\left(\frac{1}{\mu}\right) \sum_{i=2}^{n} \sum_{j=1}^{i-1} p_{i}\left|y_{i}-y_{j}\right| p_{j}$ 
educación preescolar igual a 3 , y3 a 6 , y4 a 3 , y5 a 3 , y6 a 6 , por último y7 igual a 4 Donde:

EL, es el índice Gini de educación basado en la distribución de los logros escolares de toda la población. $\mu$, es el promedio de años de escolaridad de la población.

pi y pj, corresponden a las proporciones de la población con ciertos niveles de escolaridad.

yi e yj, son los años de escolaridad en diferentes niveles educativos.

$\mathrm{n}=7$, es el número de niveles educativos.

$$
\begin{aligned}
E_{L}=\left(\frac{1}{\mu}\right) & {\left[p_{2}\left(y_{2}-y_{1}\right) p_{1}\right.} \\
& +p_{3}\left(y_{3}-y_{1}\right) p_{1}+p_{3}\left(y_{3}-y_{2}\right) p_{2} \\
& +\ldots \\
& \left.+p_{7}\left(y_{7}-y_{1}\right) p_{1}+p_{7}\left(y_{7}-y_{2}\right)+\ldots+p_{7}\left(y_{7}-y_{6}\right) p_{6}\right]
\end{aligned}
$$

El desarrollo de la ecuación 1 es el siguiente:

Thomas, Wang y Fan (2001) señalan que para calcular el Gini Educativo, es mejor utilizar los años de escolaridad que otros indicadores, ya que refleja las características del capital humano de un país, además, se tiene tres ventajas al usar la fórmula anterior, la primera es que, no existen estudios individuales sobre el nivel de la educación para muchos países; la segunda, los años de escolaridad es una variable discreta, no continua como resultado de ello, la curva Lorenz tendría siete picos y tercera la curva de Lorenz se trunca a lo largo del eje horizontal debido a que en muchos países en desarrollo una gran proporción de la población es analfabeta, lo que implica que la fórmula tradicional de Gini no puede aplicarse directamente. Sin embargo, comparada con una función continua, debido a la agrupación de datos en categorías, según el Sistema de
Información de Tendencia Educativas en América Latina (SITEAL) (2005), se da una pérdida de precisión en el cálculo del índice de desigualdad e ignora la distribución de los años de escolaridad de los individuos al interno de los ciclos y/o niveles educativos. Además, Medina (2001) dice que al utilizar datos agrupados para calcular el coeficiente de Gini, se induce a la pérdida de la información debido a que los valores son substituidos por un punto dentro del intervalo o categoría; se crea sesgo debido a que se genera una subestimación por el efecto del número de grupos, esto quiere decir que mientras menor sea la cantidad de grupos la subestimación tiende a ser más grave.

\section{Desviación estándar}

La desviación estándar es una medida de dispersión para variables de razón e intervalo, de gran utilidad en la estadística descriptiva. Se define como la raíz cuadrada de la varianza y con este valor, la desviación típica es una medida cuadrática que informa de la media de distancias que tienen los datos respecto de su media aritmética, expresada en las mismas unidades que la variable.

Para conocer con detalle un conjunto de datos, no basta conocer las medidas de tendencia central, sino que es necesaria la desviación que representan los datos en su distribución respecto de la media aritmética, con el fin de tener una visión de ellos acorde con la realidad para describirlos e interpretarlos para la toma de decisiones. En el caso de México se utiliza la desviación estándar para obtener datos precisos respecto a la distribución educativa de

$$
\sigma=\sqrt{\frac{\sum_{i=1}^{7}\left(\left(Y_{j} P_{j}\right)-E_{p}\right)^{2}}{7}}
$$


cada estado según los tres periodos de estudio correspondientes.

Donde:

EP, es el promedio de educación de la población.

Yj, es el porcentaje de los años de estudio acumulados, en donde $\mathrm{Y} 1=0, \mathrm{Y} 2=3$, $\mathrm{Y} 3=9, \mathrm{Y} 4=12, \mathrm{Y} 5=15, \mathrm{Y} 6=21, \mathrm{Y} 7=25$.

$\mathrm{Pj}$, es el porcentaje de la población acumulada, por lo tanto $\mathrm{P} 1$ es el porcentaje de la población sin escolaridad, P2 es el porcentaje de la población sin escolaridad más la población con nivel de preescolar, P3 es el porcentaje de la población $\mathrm{P} 1+\mathrm{P} 2$ más la población con educación primaria, etcétera.

\section{Curva de Kuznets}

Para rectificar las desigualdades en las observaciones de educación derivadas del Coeficiente de Gini, se obtiene una representación gráfica denominada Curva de Kuznets, con el objeto de demostrar que en las fases iníciales del crecimiento la distribución en educación será menos equitativa y solo más tarde se alcanzará la equidad esperada.

La curva de Kuznets es una representación gráfica de la hipótesis planteada por

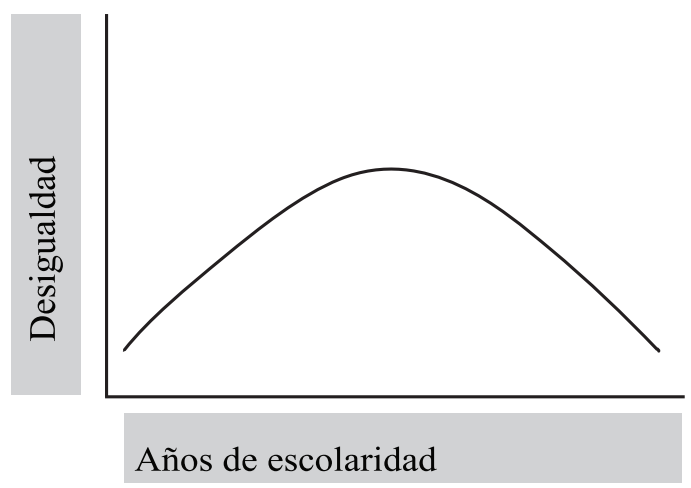

Figura 2. Curva de Kuznets Fuente: Elaboración propia.
Simón Kuznets (1955), donde establece que la desigualdad económica se incrementa a lo largo del tiempo durante el período de desarrollo de un país, posteriormente, tras cierto tiempo crítico donde el promedio de ingresos se ha alcanzado, esta curva forma una $\mathrm{U}$ invertida que comienza a decrecer, tal como se representa en la figura 2 .

Conforme al diagrama de la curva de Kuznets de la figura 2 se presenta una $\mathrm{U}$ invertida aunque las variables sobre los ejes a veces son cambiadas con desigualdad o el Coeficiente de Gini en el "eje Y”, y por desarrollo económico, y tiempo o ingreso per cápita en el "eje X".

Una de las hipótesis acerca de por qué ocurre esto, establece que en las etapas iniciales del desarrollo, cuando la inversión en capital físico es el mecanismo principal para el crecimiento económico, la desigualdad es lo que incentiva el crecimiento al trasladar recursos a quienes hacen mayor inversión y ahorro.

En contraste, en países desarrollados la adquisición de capital humano o un costo estimado en el que se ha incurrido, pero aun no se ha pagado, toma el lugar del capital físico como la mayor fuente de crecimiento y el aumento de la desigualdad se vuelve más lento al permitir que bajen los estándares de educación dado que la gente de escasos recursos no tendrá modo de financiar su educación en mercados imperfectos de crédito.

\section{Análisis de resultados}

El coeficiente de Gini obtenido refleja de forma precisa la distribución educativa de los siete niveles de escolaridad en los tres periodos de estudio (1995, 2000 y 2005) en cada Estado de la República Mexicana, el objetivo fue observar el comportamiento por cada periodo de los años acumulados de estudio de la población en función de la línea de equidistribución; posteriormente de acuerdo con la desagregación de la proporción de 
la población sin estudios y con ellos por el porcentaje de años de estudio individuales en cada categoría, se obtuvieron datos que indican el avance o retroceso de la desigualdad para cada entidad federativa. También se analizaron los porcentajes de la desviación estándar al 95\% de significancia con respecto al total de analfabetas y estudiantes en cada nivel educativo, para mostrar que en cada una de las entidades federativas el total de años de estudio de la población es mayor de un periodo a otro y obtener el porcentaje de años de estudio que cada individuo puede alcanzar. Finalmente, considerando la Curva de Kuznets como evidencia empírica se tomaron los índices de Gini obtenidos con los años de escolaridad para observar si el comportamiento de cada curva indicaba que en las etapas iniciales de crecimiento en 1995 hubo una distribución menos equitativa que en el periodo más reciente 2005 y ver además si ambas variables estaban negativamente asociadas.

\section{El Gini de educación}

De acuerdo con la desagregación de la proporción de la población sin estudios y con estudios de preescolar, primaria, secundaria, bachillerato, licenciatura y de postgrado, por el porcentaje de años de estudio individuales en cada categoría, en función del promedio de educación de cada Entidad Federativa, se obtuvieron los siguientes índices de Gini para los períodos 1995, 2000 y 2005.

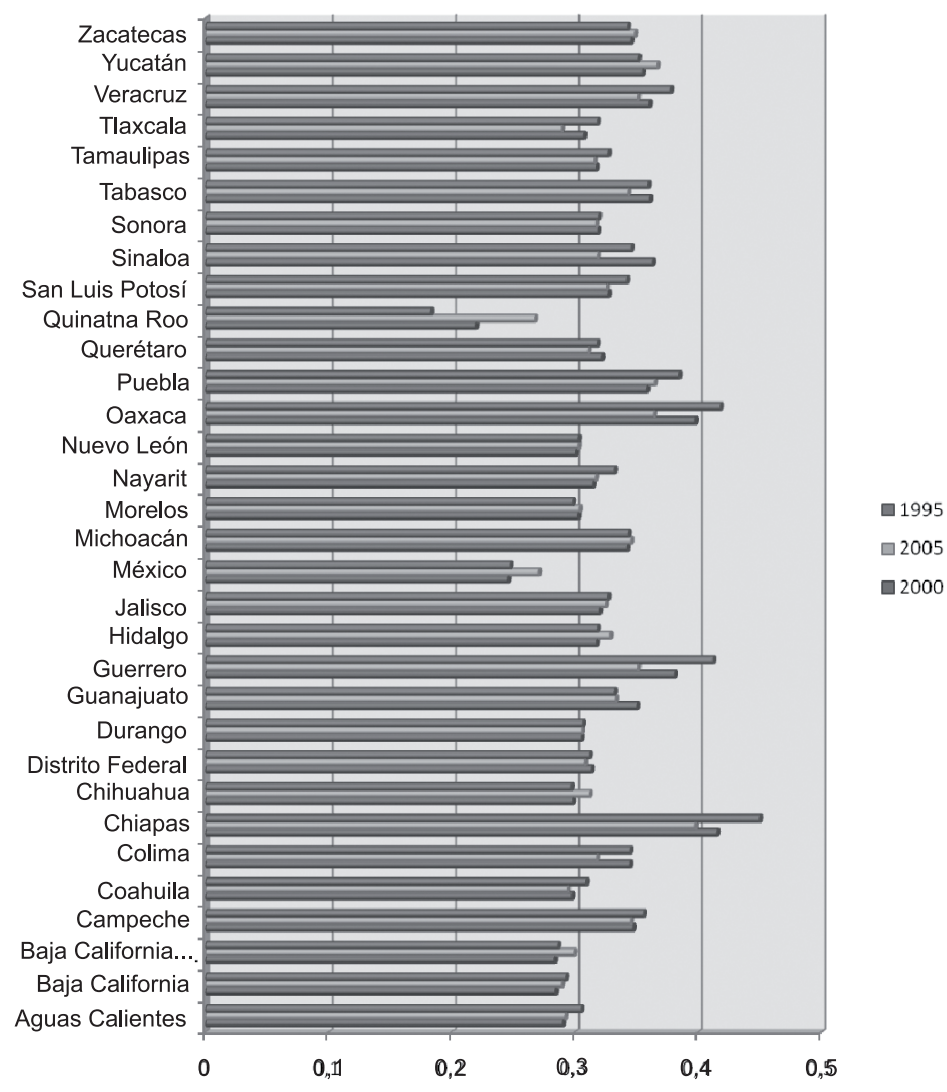

Figura 3. Índice de Gini por Entidad Federativa Fuente: Elaboración propia. 
Según los índices de desigualdad que se muestran en la figura 3, en su mayoría las entidades federativas tienden a mejorar su distribución en educación en 2005; tal es el caso de Campeche, Coahuila, Colima, Chiapas, Distrito Federal, Guanajuato, Guerrero, Oaxaca, Querétaro, San Luís Potosí, Sinaloa, Tabasco, Tamaulipas, Tlaxcala y Veracruz. Los Estados de Aguascalientes, Baja California, Durango, Jalisco, Morelos, Nayarit, Nuevo León, Puebla y Sonora, tendieron a disminuir sus índices de desigualdad con respecto a 1995 y se mantuvieron constantes para los años 2000 y 2005.

Tenemos los estados con mayores niveles de desigualdad educativa para 2005 fueron Chiapas, Puebla, Oaxaca y Guerrero; y las de menor desigualdad fueron Quintana Roo y el Estado de México. Por último, los que aumentaron sus índices de desigualdad, fueron: Baja California Sur, Chihuahua, Hidalgo, Michoacán, Estado de México, Quintana Roo, Yucatán y Zacatecas, puesto que sus índices de desigualdad se incrementaron en el $2000 \mathrm{y}$ el 2005 respecto a 1995.

\section{Estimación de la desviación estándar}

Dados los porcentajes de desviaciones estándar obtenidos, se multiplicó la desviación estándar ponderada por el porcentaje de la población en cada uno de los siete niveles de escolaridad con respecto al total de analfabetas y estudiantes en cada nivel educativo, cuyo resultado muestra que en cada una de las entidades federativas el total de años de estudio de la población es mayor de un periodo a otro, por ejemplo, en el Distrito Federal una persona tenía la probabilidad de acumular 15.36 años de estudio en 1995, en el 2000, 16.21 , y en 2005 17.33, como se puede observar en la tabla 5, es decir, en 1995 el individuo podría cursar hasta la preparatoria y en el 2005 hasta la licenciatura.

Tabla 5

Límites de distribución estándar al 95\% de significancia

\begin{tabular}{lcccccc}
\hline Entidad Federativa & $\begin{array}{c}\text { Desviación } \\
\text { estándar } \\
1995\end{array}$ & $\begin{array}{c}\text { límite } \\
\text { superior } \\
1995\end{array}$ & $\begin{array}{c}\text { Desviación } \\
\text { estándar } \\
2000\end{array}$ & $\begin{array}{c}\text { límite } \\
\text { superior } \\
2000\end{array}$ & $\begin{array}{c}\text { Desviación } \\
\text { estándar } \\
2005\end{array}$ & $\begin{array}{c}\text { límite } \\
\text { superior } \\
2005\end{array}$ \\
\hline Aguascalientes & 6.21 & 12.16 & 6.75 & 13.23 & 7.67 & 15.03 \\
Baja California & 6.81 & 13.34 & 7.05 & 13.82 & 7.79 & 15.26 \\
Baja California Sur & 6.8 & 13.32 & 7.25 & 14.21 & 7.76 & 15.2 \\
Campeche & 5.54 & 10.86 & 6.14 & 12.03 & 6.84 & 13.4 \\
Coahuila & 6.65 & 13.03 & 7.29 & 14.3 & 7.89 & 15.46 \\
Colima & 6.01 & 11.77 & 6.51 & 12.76 & 7.35 & 14.41 \\
Chiapas & 4.06 & 7.95 & 4.71 & 9.23 & 5.21 & 10.21 \\
Chihuahua & 6.27 & 12.29 & 6.67 & 13.08 & 7.19 & 14.09 \\
Distrito Federal & 7.84 & 15.36 & 8.27 & 16.21 & 8.84 & 17.33 \\
Durango & 5.81 & 11.38 & 6.32 & 12.38 & 6.95 & 13.61 \\
Guanajuato & 4.98 & 9.77 & 5.43 & 10.64 & 6.24 & 12.24 \\
Guerrero & 4.74 & 9.28 & 5.36 & 10.5 & 5.91 & 11.58 \\
Hidalgo & 5.09 & 9.98 & 5.66 & 11.09 & 6.43 & 12.59
\end{tabular}




\begin{tabular}{|c|c|c|c|c|c|c|}
\hline Entidad Federativa & $\begin{array}{c}\text { Desviación } \\
\text { estándar } \\
1995\end{array}$ & $\begin{array}{l}\text { límite } \\
\text { superior } \\
1995\end{array}$ & $\begin{array}{c}\text { Desviación } \\
\text { estándar } \\
2000\end{array}$ & $\begin{array}{l}\text { límite } \\
\text { superior } \\
2000\end{array}$ & $\begin{array}{c}\text { Desviación } \\
\text { estándar } \\
2005\end{array}$ & $\begin{array}{c}\text { límite } \\
\text { superior } \\
2005\end{array}$ \\
\hline Jalisco & 5.94 & 11.63 & 6.45 & 12.64 & 7.19 & 14.1 \\
\hline Estado de México & 6.57 & 12.87 & 7.08 & 13.87 & 7.61 & 14.91 \\
\hline Michoacán & 4.94 & 9.69 & 5.44 & 10.65 & 5.93 & 11.63 \\
\hline Morelos & 6.25 & 12.26 & 6.66 & 13.06 & 7.29 & 14.29 \\
\hline Nayarit & 5.65 & 11.08 & 6.21 & 12.16 & 7.02 & 13.77 \\
\hline Nuevo León & 7.16 & 14.02 & 7.62 & 14.93 & 8.34 & 16.35 \\
\hline Oaxaca & 4.29 & 8.41 & 4.86 & 9.53 & 5.48 & 10.75 \\
\hline Puebla & 5.21 & 10.22 & 5.82 & 11.4 & 6.33 & 12.4 \\
\hline Querétaro & 5.82 & 11.41 & 6.59 & 12.92 & 7.33 & 14.37 \\
\hline Quintana Roo & 6.17 & 12.09 & 6.85 & 13.43 & 7.44 & 14.59 \\
\hline San Luis Potosí & 5.46 & 10.71 & 5.98 & 11.72 & 6.79 & 13.3 \\
\hline Sinaloa & 5.98 & 11.72 & 6.37 & 12.49 & 7.44 & 14.57 \\
\hline Sonora & 6.62 & 12.97 & 7 & 13.71 & 7.73 & 15.16 \\
\hline Tabasco & 5.49 & 10.76 & 6.08 & 11.92 & 6.99 & 13.7 \\
\hline Tamaulipas & 6.36 & 12.46 & 6.89 & 13.5 & 7.52 & 14.75 \\
\hline Tlaxcala & 6.03 & 11.82 & 6.56 & 12.86 & 7.22 & 14.16 \\
\hline Veracruz & 5.07 & 9.94 & 5.59 & 10.95 & 6.17 & 12.1 \\
\hline Yucatán & 5.34 & 10.47 & 5.83 & 11.42 & 6.53 & 12.79 \\
\hline Zacatecas & 4.99 & 9.78 & 5.48 & 10.74 & 6.19 & 12.12 \\
\hline
\end{tabular}

Fuente: Elaboración propia.

La tabla 5 fue elaborada tomando en cuenta la distribución normal para calcular el grado de años que puede tener cada Entidad Federativa con un $95 \%$ de confiabilidad, por lo tanto, la desviación estándar fue multiplicada por 1.96 para obtener el máximo de años de estudios que puede alcanzar cada individuo en los tres periodos de estudio.

Para el caso de Chiapas, en 1995 la probabilidad el total de años acumulados por una persona podían ser de 7.95, en 2000 de 9.23 y en 2005 hasta 10.21 , es decir, que partiendo de un periodo de diez años para 1995 el individuo tendría posibilidad de cursar hasta la primaria y hasta 2005 hubiese tenido más probabilidades de terminar de cursar la secundaria. En la figura 4 , se desglosa este comportamiento para Chiapas.

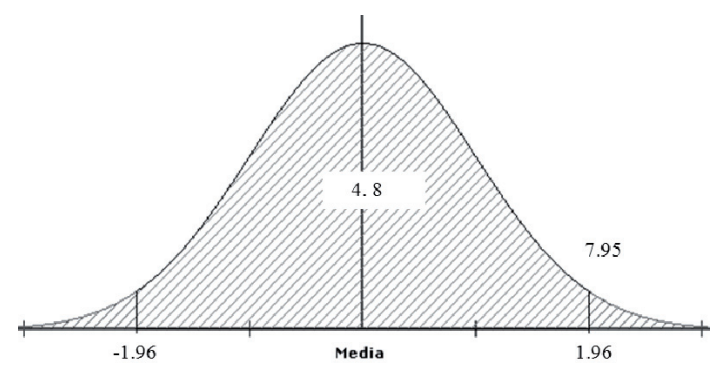

Figura 4. Desviación estándar de Chiapas (1995) Fuente: Elaboración propia. 
En el caso de Chiapas cuya distribución educativa a nivel nacional es la menor, se obtuvo una desviación estándar de 4.06 en 1995 con una alta dispersión generada por una gran variación que alcanza un máximo de 7.95 de escolaridad en el punto más alto partiendo de una media de 4.8 años de educación promedio, es decir, que el $95 \%$ de la población se encuentra entre estos años de escolaridad.

En el caso de Chiapas cuya distribución educativa a nivel nacional es la menor, se obtuvo una desviación estándar de 4.06 en 1995 con una alta dispersión generada por una gran variación que alcanza un máximo de 7.95 de escolaridad en el punto más alto partiendo de una media de 4.8 años de educación promedio, es decir, que el $95 \%$ de la población se encuentra entre estos años de escolaridad.

Si se quiere saber cuál era la probabilidad de que un chiapaneco tuviese la media de escolaridad de 9.2 años (Distrito Federal), la distribución de dichos años se encontraría a 4.4 años de la media de Chiapas, entonces, esos 4.4 años se encontraban a 2.738 desviaciones estándar de la media de Chiapas y por lo tanto la probabilidad de que este estado alcanzara el promedio del Distrito Federal era de 0.32\%.

\section{La curva de Kuznets como evidencia empírica}

Para elaborar la representación gráfica de las curvas de Kuznets, se tomaron los índices de Gini (Gini-Edu) en el "eje Y", y los años de escolaridad de cada nivel de estudios en el "eje X", para observar si el comportamiento de cada curva indicaba que en las etapas iniciales de crecimiento
(1995) se observa una distribución menos equitativa que en el periodo más reciente (2005); además si ambas variables estaban negativamente asociadas.

En los gráficos de los años 1995, 2000 y 2005 se muestra la relación entre el promedio de años de estudio y la desigualdad expresada en el coeficiente de Gini de educación. Se observa que las dos variables están negativamente relacionas, lo que indica que cuando el promedio de años de estudio en una entidad federativa es bajo, típicamente una pequeña parte de la población tiene todos los años de escolaridad concluidos, en lugar de que cada uno compartiera una pequeña pero igual cantidad de años de escolaridad, estos resultados son iguales a los encontrados por (Checchi, 2001; Thomas et al., 2001)

En el comportamiento de estas dos variables para el caso de México se encontraron dos tipos de comportamiento, uno positivo al haber entidades que tienen bajos índices de Gini, es decir una buena distribución en educación, acompañada de altos niveles educativos como en el caso del Distrito Federal, Nuevo León, Baja California y Baja California Sur, otro con altos índices de Gini y bajos niveles educativos en las entidades del Sureste de México.

Entre 1995 y 2005 hubo cambios importantes, respecto del avance de la acumulación de años de escolaridad como en el caso de Nuevo León que en ese periodo alcanzó una distribución educativa con un nivel promedio similar al del Distrito Federal.

La estimación empírica de la curva de Kuznets para 1995, con el paquete econométrico Eviews 6.1 (2008) dio el siguiente resultado, donde se establece la relación matemática entre el índice de Gini y la escolaridad:

$$
\begin{aligned}
& \text { GINI1995 }=0.697-0.0185 \text { ESCOLARIDAD }{ }^{2}+.0015 \text { ESCOLARIDAD }^{3} \\
& t \text {-estadistica } \quad(8.96) \quad(-3.90) \\
& R^{2}=0.572 \quad n=32 \quad \sigma=.033
\end{aligned}
$$


De la ecuación 3 se obtuvo la siguiente figura que muestra la relación inversa entre el índice de Gini y la escolaridad, que evidencia que niveles altos de desigualdad están relacionados con baja escolaridad y baja desigualdad con alta escolaridad.

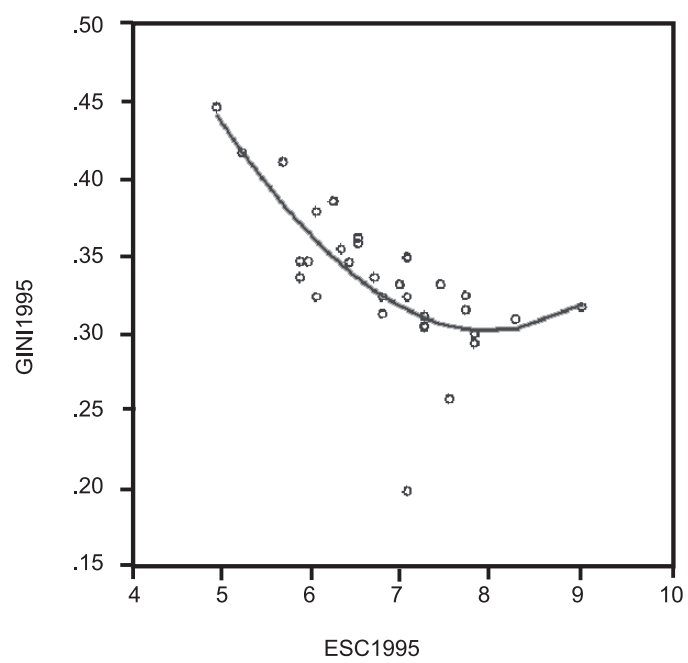

Figura 5. Curva de Kuznets, 1995

Fuente: Elaboración propia.

En la figura 5 se puede observar una pendiente negativa que nos indica una relación inversa entre el índice de Gini y la escolaridad es decir, que niveles bajos del índice están asociados a niveles más altos de escolaridad, con un punto de inflexión en 4.1 años de escolaridad y un mínimo en 8.2 años de escolaridad.

La estimación empírica de la curva de Kuznets para el 2000, dio el siguiente resultado, donde se establece la relación matemática entre el índice de Gini y la escolaridad, a través de un polinomio de tercer grado:

\footnotetext{
GINI $2000=0.696-0.0158$ ESCOLARIDAD ${ }^{2}+.0012$ ESCOLARIDAD $^{3}$ $t$-estadistica $\quad(8.69)(-3.79)$

$R^{2}=0.607 \quad n=32 \quad \sigma=.026$
}

De la ecuación 4 se obtuvo el siguiente gráfico en donde se ve la relación inversa entre el índice de Gini y la escolaridad, ya que niveles altos de desigualdad están relacionados con baja escolaridad y baja desigualdad con alta escolaridad.

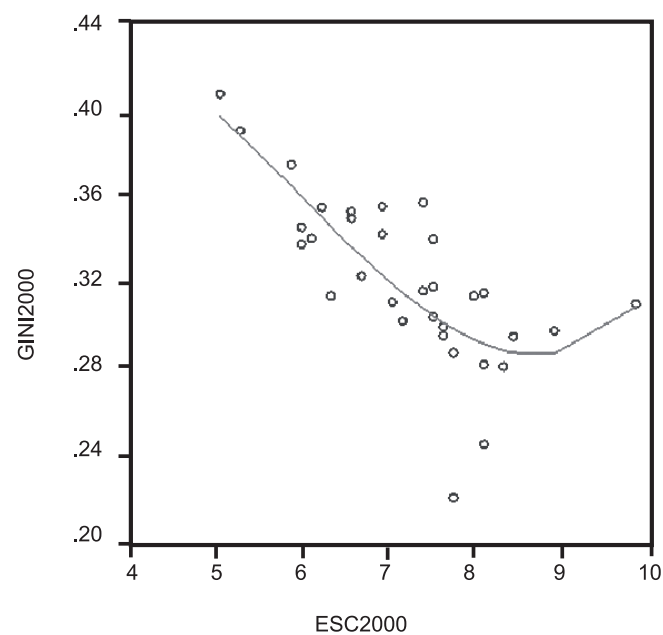

Figura 6. Curva de Kuznets, 2000

Fuente: Elaboración propia.

En la figura 6 se puede observar una pendiente negativa que nos indica una relación inversa entre el índice de Gini y la escolaridad es decir, que niveles bajos del índice están asociados a niveles más altos de escolaridad, con un punto de inflexión en 4.3 años de escolaridad y un mínimo en 8.7 años de escolaridad.

La estimación empírica de la curva de Kuznets para el 2005, dio como resultado, la relación matemática entre el índice de Gini y la escolaridad, de un polinomio de tercer grado:

\footnotetext{
GINI $2005=0.619-0.0103 E S C O L A R I D A D^{2}+.0007 E S C O L A R I D A D^{3}$ $t$-estadística (89.85) $(-3.69)$ (3.16) 5 $R^{2}=0.681 \quad n=32 \quad \sigma=.017$
}

De la ecuación 5 se obtuvo el siguiente gráfico que muestra la relación inversa entre el índice de Gini y la escolaridad, debido a que niveles altos de desigualdad están relacionados con baja escolaridad y baja desigualdad con alta escolaridad. 


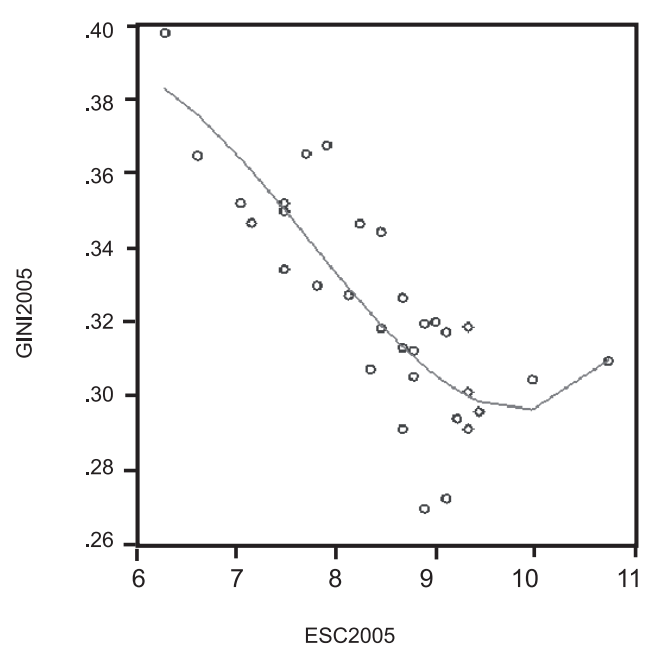

Figura 7. Curva de Kuznets, 2005

Fuente: Elaboración propia

Se puede observar en esta figura 7, una pendiente negativa que nos indica una relación inversa entre el índice de Gini y la escolaridad es decir, que niveles bajos del índice están asociados a niveles más altos de escolaridad, con un punto de inflexión en 6.3 años de escolaridad y un mínimo en 9.8 años de escolaridad.

Lo que se observa en los tres gráficos anteriores es que a través del tiempo las curvas de Kuznets se desplazan hacia abajo debido a que la pendiente o velocidad de descenso se hace más lenta, los puntos de inflexión y los valores mínimos se desplazan hacia la derecha, los puntos de inflexión que nos señala cuando la curva cambia de sentido para convertirse de cóncava a convexa quiere decir que los años de escolaridad tiende a ser mayores para que se inicie un cambio de sentido de las pendientes, los mínimos nos indican cuando la pendiente es cero, para posteriormente cambiar a positiva.

\section{Conclusión general}

Se tienen fuertes esperanzas en las posibilidades de que la educación sea un motor para mejorar las condiciones de vida de la población puesto que funge como una de las piezas claves para erradicar la pobreza de acuerdo con los hallazgos de este estudio se constató que la educación en México es un fenómeno creciente, ya que el promedio en años de escolaridad para 1995 fue de 7.0, en el 2000 de 7.6 y en el 2005 de 8.2 , esto indicaría un avance de 1.2 años de escolaridad, sin embargo, su distribución sigue siendo altamente desigual ya que los niveles de concentración educación posbásica se encuentran en estratos superiores de las entidades federales con mejor distribución de educación, lo que ha provocado un lento crecimiento de la escolaridad principalmente para los estados del sureste del país, como Chiapas. Esos bajos perfiles de escolaridad en todos los estratos sociales son un reflejo de la urgente necesidad por atender simultáneamente el crecimiento en la educación básica y posbásica.

La aplicación de las técnicas de distribución de la educación que se utilizaron reflejan el atraso educativo que presenta el país y la poca perspectiva a futuro que se tiene para incrementar los niveles educativos, por lo que es necesario que el gobierno diseñe una estrategia que permita involucrar a cada individuo con los medios de desarrollo que la educación puede darle, adecuados a sus necesidades y capacidades de aprendizaje para que además de surtir efectos positivos en cada entidad federativa tengan un efecto en la productividad y competitividad de la economía nacional, así como en el bienestar individual.

Una buena educación es condición necesaria pero no suficiente para que los países prosperen, como se puede deducir de las entidades más ricas que tienen mejores niveles de educación y sobre todo mayor educación promedio, como es el caso del Distrito Federal y los estados de Norte de México, porque si los proyectos que generan riqueza no son rentables, difícilmente llegarán las tecnologías y las inversiones necesarias para que la población educada se inserte en ellos. Sin incentivos e instituciones 
adecuadas una buena educación no rendirá los frutos esperados, por lo que además de las estrategias necesarias para mejorar la educación es importante una utilización transparente y óptima de los recursos destinados a ella.

El reto del país es lograr una educación inclusiva y de calidad por ser esta la clave fundamental para el desarrollo individual y social que nos hace partícipes de un sistema económico, político, social y cultural cada vez mejor. De acuerdo con la metodología y hallazgos de la investigación descrita es indispensable mejorar la distribución de la educación y la dispersión, lo cual se mostró con el comportamiento del índice de Gini, la desviación estándar y la curva de Kuznets, destinando más recursos humanos y monetarios a aquellos estados donde las oportunidades son más escasas y la distribución educativa desigual como es el caso del sureste del país, en virtud de haber mostrado la población de estos estados menores promedios educativos $\mathrm{y}$ pocos estudiantes en los niveles altos, lo que implica que se deben igualar las condiciones educativas en México para favorecer el desarrollo económico y social.

\section{Referencias bibliográficas}

Banco Interamericano de Desarrollo BID (1999.) América Latina Frente a la Desigualdad. Informe de Progreso Económico y Social. Washington D. C.: BID.

Barro, R. (2000). Inequality and Growth in a Panel of Countries, Journal of Economic Growth, 5(1), 87-120.

Barro, R. \& Lee, J.W. (1993). International comparisons of educational attainement, Journal of Monetary Economics, (32), 363-394.

Becker, G. (1967). Human Capital and the Personal Distribution of Income: An Analytical Approach. Woytinsky Lecture no.1, Institute of Public
Administration, University of Michigan.

Becker, G. (1983). El capital humano. México DF: Alianza Editorial.

Birdsall, N. \& Londoño, J. L. (1997). Asset Inequality Matters: An Assesment of the World Bank's Approach to Inequality Reduction. The American Economic Review. 87, (2), 32-37.

Checchi, D. (2001). Education, Inequality and Income Inequality. Distributional Analysis Research Programme, Discussion Paper no. DARP 52. London: STICERD, London School of Economics.

Deaton, A. (1997). Analysis of Household Surveys. Baltimore MD: Johns Hopkins University Press.

Debraj, R. (1998). Development Economics. Princeton, New Jersey: Princeton University Press.

Eviews 6.1. (2008). Paquete estadístico de Quantitative Micro Software (QMS)

Krugman, P. (2008). Trade and wages, reconsidered. Brookings Papers on Economic Activity (1) 103-154.

Kuznets, S. (1955). Economic Growth and Income Inequality. American Economic Review 45 (1), 1-28.

Lim, A. S. K. \& Tang, Kam K. (2008). Human Capital Inequality and The Kuznets Curve The Developing Economies, 46(1), 26-51.

López, R. Thomas, V. \& Wang, Y. (1999). Addressing the Education Puzzle: The Distribution of Education and Economic Reforms. Policy Research Working Papers. (2031). Whasington D.C.: World Bank .

Medina, F. (2001). Consideraciones sobre el Índice de Gini para la Concentración del Ingreso. Serie de Estudios Estadisticos y Prospectivos, (9) Santiago de Chile: CEPAL.

Organización para la Cooperación y el Desarrollo Económicos OCDE. (2007) Education at a Glance. Recuperado de http://www.oecd.org/ 
document /30/0,3746,en_2649_39263 238_39251550_1_1_1_1,00.html

Organización para la Cooperación y el Desarrollo Económicos OCDE. (2010) México, Recuperado de http://www. oecd.org/document/6/0,3746,es_ 36288966_36287974_44467078_1_ _1_1,00.html, 22/10/2010

Organización para la Cooperación y el Desarrollo Económicos OCDE. (2011). Panorama de la Educación en México. Recuperado de http://www.oecd.org/ dataoecd/32/32/48667648.pdf

Ram, R. (1989). Level of Development and Income Inquality: An Extension of Kuznets-Hypothesis to the World Economy. Kyklos; 42(1), 73-79

Salgado, J. (1983). La curva de Lorenz y el Índice de Gini. Revista Contradicción, Facultad de Economía de la Universidad Autónoma del Estado de México, 8-9 (2), 44-52.

Schultz T. P. (1993). Investments in the Schooling and Health of Women and Men: Quantities and Returns. The Journal of Human Resources 28 (4), 694-734.

Sistema de Información de Tendencia Educativas en América Latina SITEAL. (2005). Medidas de desigualdad para variables educativas. Boletín (4). Santiago de Chile.
Thomas, V., Wang, Y. \& Fan, X. (2001). Measuring Education Inequality: Gini Coefficients of Education. World Bank Policy Research Working Paper, (WPS2525). Washington, D.C.: World Bank.

\section{Fuentes de Datos}

Instituto Nacional de Estadística, Geografía e Informática de México, INEGI (1998). Anuario de Estadísticas por Entidad Federativa. México DF.

Instituto Nacional de Estadística, Geografía e Informática de México, INEGI (2003). Anuario de Estadísticas por Entidad Federativa. México DF.

Instituto Nacional de Estadística, Geografía e Informática de México, INEGI (2008). Anuario de Estadisticas por Entidad Federativa. México DF.

Instituto Nacional de Evaluación Educativa, INEE (2007). Panorama Educativo de México Indicadores del Sistema Educativo Nacional 2007. Recuperado de http://www. inee.edu.mx/images/stories/ Publicaciones/Panorama_educativo/2007/Completo/panorama2007completoa.pdf 